\title{
Florística e síndromes de dispersão de espécies arbustivo-arbóreas no Parque Estadual Mata São Francisco, PR, Brasil ${ }^{1}$
}

\author{
Maristela Yuka Zama ${ }^{2,4}$, Yves Rafael Bovolenta ${ }^{3}$, Eloísa de Souza Carvalho ${ }^{3}$, Diego Resende Rodrigues ${ }^{3}$, \\ Carla Gomes de Araujo², Maria Aparecida da Fonseca Sorace² e Diego Gimenes Luz ${ }^{2}$
}

Recebido: 17.08.2011; aceito: 18.07.2012

\begin{abstract}
Floristic composition and diaspore dispersal syndromes of shrubs and tree species in Parque Estadual Mata São Francisco, Paraná State, Brazil). The present study aimed to describe the floristics and strategies of propagule dispersal, as well as the types of fruits from woody species in a semi-deciduous forest fragment, at Parque Estadual Mata São Francisco, which had suffered selective logging and became a conservation area in 1994. The survey was conducted monthly from October/2007 to July/2009 considering shrubs and woody individuals with DBH $\geq 5 \mathrm{~cm}$, resulting in the identification of 99 species and 77 genera distributed in 36 botanical families among the dispersion syndromes, zoochory was the most frequent, represented by $64 \%$ of the species, followed by anemochory $(23 \%)$, and autocory $(13 \%)$. The most abundant fruit was the capsule type. Comparisions with a previous study made in the same area, highlighted an increase of richness, high turnover of species and higher proportion of dispersed animal species, suggesting a successional advancement of this fragment.

Key words: Atlantic Forest, semi-deciduous seasonal forest, southern Brazil, zoochorics species
\end{abstract}

RESUMO - (Florística e síndromes de dispersão de espécies arbustivo-arbóreas no Parque Estadual Mata São Francisco, PR, Brasil). O presente estudo teve por objetivos descrever a florística e as estratégias de dispersão de propágulos, assim como os tipos de frutos das espécies arbustivo-arbóreas em um fragmento de Floresta Estacional, no Parque Estadual Mata São Francisco, onde ocorreu extração seletiva de madeira até se tornar Unidade de Conservação em 1994. O levantamento florístico foi realizado durante os meses de outubro/2007 a julho/2009, considerando os indivíduos arbustivos e arbóreos com DAP $\geq 5 \mathrm{~cm}$, resultando na identificação de 99 espécies e 77 gêneros, distribuídos em 36 famílias botânicas. A síndrome de dispersão mais frequente foi a zoocoria com 64\% das espécies, seguida pela anemocoria (23\%), e autocoria (13\%); e o fruto mais abundante foi do tipo cápsula. As comparações com estudo anterior desenvolvido na área, ressaltaram o aumento na riqueza, grande substituição de espécies e maior proporção de espécies zoocóricas, sugerindo o avanço sucessional desse fragmento.

Palavras-chave: espécies zoocóricas, floresta estacional semidecidual, Mata Atlântica, sul do Brasil

\section{Introdução}

A Mata Atlântica é considerada um dos biomas mais ricos em biodiversidade do planeta e também um dos mais ameaçados de extinção (Fundação SOS Mata Atlântica \& INPE 2008), ocupando 17 Estados brasileiros além de áreas na Argentina e no Paraguai (Fundação SOS Mata Atlântica \& INPE 2008). Estima-se que sua área original foi reduzida para cerca de 11,4 a 16\% em remanescentes fragmentados, dos quais $80 \%$ apresentam áreas menores que 50 ha (Ribeiro et al. 2009).
Esse bioma possui no Estado do Paraná três grandes classes de formações florestais: Floresta Ombrófila Densa, Floresta Ombrófila Mista e Floresta Estacional Semidecidual (Veloso et al. 1991). A Floresta Estacional Semidecidual possui fisionomia marcada pela estacionalidade e semidecidualidade foliar (Veloso et al. 1991, Mikich \& Silva 2001) e encontra-se em área de dupla estacionalidade, ou seja, uma estação de clima tropical com chuvas intensas no verão e outra subtropical, com ausência de período seco (Veloso et al. 1991). No Paraná, essa formação florestal ocorre no oeste, noroeste e norte

1. Parte do Trabalho de Conclusão de Curso da Primeira Autora

2. Universidade Estadual do Norte do Paraná, Departamento de Biotecnologia, 86360-000 Bandeirantes, PR, Brasil

3. Universidade Estadual de Londrina, Departamento de Biologia Animal e Vegetal, 86051-990 Londrina, PR, Brasil

4. Autor para correspondência: maristela_zama@hotmail.com 
do Estado, em grande parte coincidindo com a região de ocorrência da popular "terra roxa", solo de origem basáltica com alta fertilidade (Mikich \& Silva 2001).

A Floresta Estacional Semidecidual é considerada a segunda formação com maior número de espécies listadas em trabalhos de fitossociologia, tendo sido registrados 272 taxa (Isernhagen 2003). Segundo Campassi (2006), o ecossistema apresenta composição florística intermediária entre a Floresta Ombrófila e a Floresta Estacional Decidual, sendo que a primeira possui maior proporção de dispersão zoocórica, com frutos mais vistosos, enquanto a segunda, maior dispersão abiótica, devido à característica vegetacional apresentada.

Um estudo fitossociológico realizado entre 1994 e 1995 apontou grande diversidade de espécies arbóreas na Floresta Estacional Semidecidual do Parque Estadual Mata São Francisco (PEMSF). O fragmento sofreu exploração de madeira, e a partir de 1994, tornou-se unidade de proteção integral, sendo atualmente a maior Unidade de Conservação do norte do Paraná, cuja importância é relevante tanto para a preservação de espécies e educação ambiental, quanto para a pesquisa científica (Tomé et al. 1999). Porém, além da composição florística também é necessário conhecer alguns aspectos ecológicos, como a dispersão de sementes das espécies que ocupam os remanescentes florestais. Tal abordagem é importante para compreender o funcionamento dessas florestas e seu processo de regeneração, sendo a dispersão o meio para controlar e manter a diversidade biológica e pré-requisito para a continuidade dos ecossistemas (Mikich \& Silva 2001).

O padrão de dispersão de sementes não só determina a área potencial de recrutamento das plantas, mas também serve como base para os processos subsequentes, tais como a competição, predação e reprodução (Nathan \& Muller-Landau 2000). A dispersão também afeta o fluxo de genes $\mathrm{e}$, portanto, influencia a estrutura genética dentro e entre populações (Ouborg et al. 1999). A estrutura, morfologia, entre outras características apresentadas pelo fruto, permite que as plantas sejam dispersas com maior facilidade por seu determinado agente dispersor (Van der Pijl 1982). O conjunto dessas características ajuda a determinar a síndrome de dispersão das espécies (Howe \& Smallwood 1982).

O conhecimento florístico das florestas tropicais e o estudo de interações ecológicas como a dispersão de sementes constitui importante ferramenta para a conservação ajudando a compreender a estrutura e a dinâmica das comunidades e o seu processo de regeneração (Howe \& Smallwood 1982, Kinoshita et al.2006). Nesse contexto, os objetivos do presente estudo foram analisar a composição florística, investigar a ocorrência e a distribuição das síndromes de dispersão de frutos ou sementes, assim como os tipos de frutos das espécies arbustivo-arbóreas encontrados no Parque Estadual Mata São Francisco e relacioná-los com o levantamento florístico realizado por Tomé et al. (1999) no mesmo fragmento, para se ter uma avaliação do processo sucessional nesse período.

\section{Material e métodos}

Área de estudo - O Parque Estadual Mata São Francisco (PEMSF), criado pelo Decreto-Lei no 4333 de 05 de dezembro de 1994, localiza-se no norte do Estado do Paraná, entre os municípios de Santa Mariana e Cornélio Procópio, latitude $23^{\circ} 09^{\prime} 55^{\prime \prime S}$ e longitude $50^{\circ} 33^{\prime} 93^{\prime \prime W}$ (centro do fragmento) (Tomé et al. 1999) e apresenta área de 832,58 hectares, classificada como Floresta Estacional Semidecidual. Atualmente o remanescente é cercado por culturas vegetais, principalmente de grãos, e até se tornar Unidade de Conservação, ocorreu a retirada desordenada de madeira, palmito e queimadas, a qual possivelmente resultou no excesso de trepadeiras e bambus (Tomé \& Vilhena 1996).

O clima da região, segundo a classificação de Köppen (1948), caracteriza-se como Cfa, com precipitação média entre 1.200 a $1.400 \mathrm{~mm}$ distribuídos irregularmente durante o ano (IAPAR 2000), temperatura média superior a $10^{\circ} \mathrm{C}$ e altitude de aproximadamente $520 \mathrm{~m}$ (Tomé et al. 1999). As unidades de solo predominantes são Latossolo Vermelho eutroférrico e Nitossolo Vermelho eutroférrico, com inclusões de Chernossolos e Gleissolos, todos considerados solos de alta fertilidade (Santos 1999).

Metodologia - O estudo foi realizado no período de outubro de 2007 a julho de 2009, no qual empregou-se o método de parcelas quadradas (Mueller-Dombois \& Ellenberg 1974), as quais mediam $20 \mathrm{~m} \times 20 \mathrm{~m}$ e distanciavam-se em $200 \mathrm{~m}$, dispostas em duas transecções contíguas no fragmento, distantes a cada $500 \mathrm{~m}$ no sentido norte-sul totalizando 21 parcelas com 0,840 ha de área amostrada. Em cada parcela foram amostrados todos os indivíduos arbustivo-arbóreos com diâmetro a $1,30 \mathrm{~m}$ de altura do solo $\geq 5 \mathrm{~cm}$. Os indivíduos encontrados em fase de frutificação, não 
inclusos dentro das parcelas, também foram incluídos nas avaliações, com a finalidade de amostrar o maior número de espécies arbustivo-arbóreas e aumentar a confiabilidade dos resultados da pesquisa.

O material botânico coletado foi herborizado e incorporado no acervo do herbário da Universidade Estadual do Norte do Paraná - campus Luiz Meneghel (CBT). As espécies foram classificadas pelo sistema APG III (2009). A identificação botânica foi realizada por meio de consultas ao herbário do Museu Botânico Municipal de Curitiba (MBM), bibliografias especializadas e consultas a especialistas.

A classificação dos frutos foi baseada nas características apresentadas: baga ou drupa quando apresentaram pericarpo carnoso; pericarpo seco deiscente - que se abre quando maduro: folículo, legume, cápsula e síliqua; pericarpo seco indeiscente - que não se abre quando maduro: aquênio, cariopse e sâmara (Ferri 1983).

As espécies foram classificadas segundo a síndrome de dispersão de acordo com Van der Pijl (1982) em três categorias: anemocóricas, zoocóricas e autocóricas.

\section{Resultados}

Foram amostradas 99 espécies nativas, pertencentes a 77 gêneros de 36 famílias botânicas (tabela 1). Quanto ao mecanismo de dispersão, as espécies foram classificadas em zoocóricas (64\%), seguidas por anemocóricas (23\%) e autocóricas (13\%) (figura 1).

Segundo os caracteres morfológicos dos frutos, das 99 espécies 30 (30\%) foram classificadas como cápsula, 27 (28\%) drupa, 19 (19\%) baga, 11 (11\%) legume, seis $(6 \%)$ sâmara, dois $(2 \%)$ folículo, dois $(2 \%)$ aquênio e dois (2\%) pseudofruto (figura 2 ).

As famílias com maior número de representantes no atual estudo foram Fabaceae (Leguminosae) subfamília Faboideae e Meliaceae, com oito e sete espécies respectivamente, sendo que a primeira apresentou frutos dos tipos sâmara e legume, enquanto a segunda tem a cápsula como fruto característico. As famílias com maior número de espécies zoocóricas foram Myrtaceae e Meliaceae (seis espécies cada).

\section{Discussão}

O total de espécies identificadas acompanha os resultados encontrados nos levantamentos realizados em Florestas Estacionais Semideciduais da região norte do Estado do Paraná, observados em Soares-Silva \& Barroso (1992) com 100 espécies amostradas, Soares-Silva et al. (1992) com 104 espécies, 92 espécies por Dolibaina et al. (1993) e 93 espécies por Costa et al. (2011), demonstrando uma riqueza expressiva de espécies.

No estudo realizado por Tomé et al. (1999) no PEMSF foram identificadas 36 famílias botânicas, abrangendo 85 espécies, sendo que dessas, cinco espécies não foram identificadas, uma não representa um gênero botânico reconhecido (Guaca sp.), sete foram identificadas até o nível de família, 16 até gênero e 56 espécies. No presente estudo, apesar de um menor esforço amostral em comparação com o inventário de Tomé et al. (1999), foi encontrada uma maior riqueza de espécies arbustivo-arbóreas. Das 99 espécies amostradas 34 não haviam sido registradas no fragmento (tabela 1).

Tomé et al. (1999) encontraram 13 espécies que não foram encontradas neste estudo: Phytolacca dioica L., Qualea sp., Guarea guidonia (L.) Sleumer, Maclura tinctoria (L.) D. Don ex Steud, Bastardiopsis densiflora (Hook. \& Arn.) Hassl., Myroxylon peruiferum L. f, Diatenopteryx sp., Allophyllus sp., Aloysia virgata (Ruiz \& Pav.) Juss., Annona cacans Warm., Cordia ecalyculata Vell., Prockia crucis P. Browne ex L., e Sebastiania commersoniana (Baill.) Smith \& Downs. Dentre essas espécies, nove são consideradas características de estágios iniciais de sucessão (pioneira ou secundária ini-cial) em estudos realizados em áreas de Floresta Estacional Semidecidual (Fonseca \& Rodrigues 2000, Silva \& Soares-Silva 2000, Aquino \& Barbosa 2009), pela classificação de Budowski (1965).

Somando-se os resultados de Tomé et al. (1999) com os resultados atuais tem-se o total de 113 espécies arbustivo-arbóreas nativas encontradas nesse fragmento. As comparações entre o presente estudo e o estudo de Tomé et al. (1999) no PEMSF caracterizam um aumento na riqueza e substituição de espécies, que segundo Gandolfi (2000) sugere o processo de sucessão ecológica e consequente ampliação da complexidade do ecossistema. Os resultados podem estar relacionados com o histórico de exploração do fragmento, uma vez que o primeiro estudo foi realizado em 1994 e 1995 (Tomé et al. 1999), período imediatamente após a área ser transformada em unidade de proteção integral, e o presente estudo foi realizado cerca de 14 anos após cessar a extração seletiva de madeira. 
Tabela 1. Florística do Parque Estadual Mata São Francisco, PR, Brasil, com a listagem das famílias botânicas, nomes científicos e nomes populares das espécies. Síndromes de dispersão (SD): Anemocoria (ane); Autocoria (aut); Zoocoria (zoo). Tipos de fruto (TF): Aquênio (A); Baga (B); Cápsula (C); Drupa (D); Folículo (F); Legume (L); Pseudofruto (P); Sâmara $(\mathrm{S})$. Grupo ecológico $(\mathrm{GE})$ : Pioneira $(\mathrm{P})$; Secundária inicial $(\mathrm{S})$; Secundária tardia $(\mathrm{T})$; Clímax $(\mathrm{C})$. Número de registro no herbário (No. CBT). *espécies encontradas apenas no estudo atual.

Table 1. Floristic composition of Parque Estadual Mata São Francisco, Paraná State, Brazil, with botanical family names, scientific names, and popular names. Dispersal syndrome (SD): Anemochory (ane); Autochory (aut); Zoochory (zoo); Fruit type (TF): Achene (A); Berry (B); Capsule (C); Drupe (D); Follicle (F); Legumen (L); Pseudo fruit (P); Samara (S); Ecological classification (GE): Pioneer (P); Initial secondary (I); Late secondary (T); Climax (C). Herbarium registration number (No. CBT). *species found only in the current study.

\begin{tabular}{|c|c|c|c|c|c|}
\hline Família/ Nome científico & Nome popular & $\mathrm{SD}$ & $\mathrm{TF}$ & GE & No. CBT \\
\hline Acanthaceae & - & - & - & - & - \\
\hline Justicia brasiliana Roth.* & justicia & aut & $\mathrm{C}$ & I & 3700 \\
\hline Anacardiaceae & - & - & - & - & - \\
\hline Astronium graveolens Jacq. & guaritá & ane & $\mathrm{B}$ & $\mathrm{T}$ & 3701 \\
\hline Annonaceae & - & - & - & - & - \\
\hline Annona sylvatica A. St.-Hil. & araticum-da-mata & zoo & $\mathrm{B}$ & I & 3702 \\
\hline Apocynaceae & - & - & - & - & - \\
\hline Aspidosperma polyneuron Müll. Arg. & peroba-rosa & ane & $\mathrm{F}$ & $\mathrm{T}$ & 3703 \\
\hline Rauvolfia sellowii Müll. Arg. & casca-d'anta & zoo & $\mathrm{B}$ & $\mathrm{T}$ & 3704 \\
\hline Tabernaemontana hystrix Steud.* & leiteiro & zoo & $\mathrm{C}$ & $\mathrm{P}$ & 3705 \\
\hline Araliaceae & - & - & - & - & - \\
\hline Schefflera morototoni (Aubl.) Maguire, Steyerm. \& Frodin & mandioqueiro & zoo & $\mathrm{D}$ & I & 3706 \\
\hline Arecaceae & - & - & - & - & - \\
\hline Euterpe edulis Mart. & palmiteiro & zoo & $\mathrm{D}$ & $\mathrm{C}$ & 3707 \\
\hline Syagrus romanzoffiana (Cham. ) Glassm. & jerivá & zoo & $\mathrm{D}$ & $\mathrm{T}$ & 3708 \\
\hline Bignoniaceae & - & - & - & - & - \\
\hline Jacaranda micrantha Cham. & jacarandá & ane & $\mathrm{C}$ & I & 3709 \\
\hline Boraginaceae & - & - & - & - & - \\
\hline Cordia trichotoma (Vell.) Arrab. Ex Steud. & louro-pardo & ane & $\mathrm{D}$ & $\mathrm{T}$ & 3710 \\
\hline Cannabaceae & - & - & - & - & - \\
\hline Celtis iguanae (Jacq.) Sargent* & esporão-de-galo & zoo & $\mathrm{D}$ & $\mathrm{P}$ & 3711 \\
\hline Trema micrantha (L.) Blume & crindiúva & zoo & $\mathrm{D}$ & $\mathrm{P}$ & 3712 \\
\hline Cardiopteridaceae & - & - & - & - & - \\
\hline Citronella sp.* & citronela & zoo & $\mathrm{B}$ & $\mathrm{T}$ & 3713 \\
\hline Caricaceae & - & - & - & - & - \\
\hline Jacaratia spinosa (Aubl.) A. DC. & jaracatiá & zoo & $\mathrm{B}$ & $\mathrm{T}$ & 3714 \\
\hline Euphorbiaceae & - & - & - & - & - \\
\hline Actinostemon concolor (Spreng.) Müll. Arg.* & laranjeira-do-mato & aut & $\mathrm{C}$ & $\mathrm{C}$ & 3715 \\
\hline Alchornea glandulosa Poepp. \& Endl. & folha-de-bolo & zoo & $\mathrm{C}$ & I & 3716 \\
\hline Alchornea triplinervia (Spreng.) Müll. Arg. & tapiá & zoo & $\mathrm{C}$ & I & 3717 \\
\hline Croton floribundus Spreng. & capixingui & aut & $\mathrm{C}$ & I & 3718 \\
\hline Sebastiania brasiliensis Spreng. & leiteiro-da-folha-fina & aut & $\mathrm{C}$ & I & 3719 \\
\hline Pachystroma longifolium (Nees.) I. M. Johnston & guacá & aut & $\mathrm{C}$ & $\mathrm{T}$ & 3720 \\
\hline Fabaceae & - & - & - & - & - \\
\hline Holocalyx balansae Micheli & alecrim-de-campinas & zoo & $\mathrm{D}$ & $\mathrm{C}$ & 3721 \\
\hline
\end{tabular}


Tabela 1 (continuação)

\begin{tabular}{|c|c|c|c|c|c|}
\hline Família/ Nome científico & Nome popular & $\mathrm{SD}$ & $\mathrm{TF}$ & GE & No. CBT \\
\hline Bauhinia forficata Link* & pata-de-vaca & aut & $\mathrm{L}$ & I & 3722 \\
\hline Centrolobium tomentosum Guillemin. ex Benth. & araribá & ane & $\mathrm{S}$ & I & 3723 \\
\hline Lonchocarpus cultratus (Vell.) H. & feijão-crú & ane & $\mathrm{L}$ & $\mathrm{I} / \mathrm{T}$ & 3724 \\
\hline Lonchocarpus muehlbergianus Hassl. & embira-de-sapo & ane & $\mathrm{F}$ & I & 3725 \\
\hline Lonchocarpus subglaucescens Mart. Ex Benth.* & timbó & ane & $\mathrm{L}$ & $\mathrm{T}$ & 3726 \\
\hline Machaerium stipitatum (DC.) Vogel & sapuvinha & ane & $\mathrm{S}$ & $\mathrm{I} / \mathrm{T}$ & 3727 \\
\hline Machaerium nyctitans (Vell.) Benth. & mau-vizinho & ane & $\mathrm{S}$ & $\mathrm{I} / \mathrm{T}$ & 3728 \\
\hline Myrocarpus frondosus Fr. Allem. & óleo-pardo & ane & $\mathrm{S}$ & $\mathrm{T}$ & 3729 \\
\hline Ormosia arborea (Vell.) Harms* & olho-de-cabra & zoo & $\mathrm{L}$ & $\mathrm{C}$ & 3730 \\
\hline Senegalia polyphylla (DC.) Britton \& Rose & monjoleiro & zoo & $\mathrm{L}$ & I & 3731 \\
\hline Anadenanthera colubrina (Vell.) Brenan & angico-branco & aut & $\mathrm{L}$ & $\mathrm{T}$ & 3732 \\
\hline Enterolobium contortisiliquum (Vell.) Morong & timburi & zoo & $\mathrm{L}$ & $\mathrm{T}$ & 3733 \\
\hline Inga marginata Willd. & ingá-de-folha-lisa & zoo & $\mathrm{L}$ & I & 3734 \\
\hline Inga striata Benth. & ingá-de-folha-peluda & zoo & $\mathrm{L}$ & $\mathrm{T}$ & 3735 \\
\hline Parapiptadenia rigida (Benth.) Brenan* & angico-vermelho & aut & $\mathrm{L}$ & $\mathrm{T}$ & 3736 \\
\hline Piptadenia gonoacantha (Mart.) J.F. Macbr. & pau-jacaré & aut & $\mathrm{L}$ & I & 3737 \\
\hline Lamiaceae & - & - & - & - & - \\
\hline Aegiphila mediterrânea Vell.* & tamanqueira & $\mathrm{zoO}$ & $\mathrm{D}$ & I & 3738 \\
\hline Lauraceae & - & - & - & - & - \\
\hline Ocotea elegans $\mathrm{Mez}$ & canela-sassafraz-do campo & zoo & $\mathrm{D}$ & I & 3739 \\
\hline Ocotea puberula (Rich.) Ness & canela-sebo & zoo & $\mathrm{D}$ & $\mathrm{T}$ & 3740 \\
\hline Endlicheria paniculata (Spreng.) J.F. Macbr & canela-de-veado & zoo & $\mathrm{D}$ & $\mathrm{C}$ & 3741 \\
\hline Nectandra megapotamica (Spreng.) Mez & canelinha & zoo & $\mathrm{D}$ & $\mathrm{T}$ & 3742 \\
\hline Malvaceae & - & - & - & - & - \\
\hline Pseudobombax grandiflorum (Cav.)A. Robyns & embiruçu & ane & $\mathrm{C}$ & $\mathrm{T}$ & 3743 \\
\hline Ceiba speciosa (A. St.-Hil.) Ravenna & paineira & ane & $\mathrm{C}$ & $\mathrm{T}$ & 3744 \\
\hline Guazuma ulmifolia Lam.* & mutambo & ane & $\mathrm{C}$ & I & 3745 \\
\hline Heliocarpus popayanensis Kunth & algodoeiro & ane & $\mathrm{C}$ & I & 3746 \\
\hline Malpighiaceae & - & - & - & - & - \\
\hline Bunchosia pallescens Scottsb.* & - & zoo & $\mathrm{B}$ & $\mathrm{T}$ & 3747 \\
\hline Melastomataceae & - & - & - & - & - \\
\hline Miconia sp. $1 *$ & - & zoo & $\mathrm{B}$ & - & 3748 \\
\hline Miconia sp. 2* & - & zoo & $\mathrm{B}$ & - & 3749 \\
\hline Miconia sp. 3* & - & zoo & $\mathrm{B}$ & - & 3750 \\
\hline Meliaceae & - & - & - & - & - \\
\hline Cabralea canjerana (Vell.) Mart. & canjarana & zoo & $\mathrm{C}$ & I & 3751 \\
\hline Cedrela fissilis Vell. & cedro & ane & $\mathrm{C}$ & $\mathrm{T}$ & 3752 \\
\hline Guarea macrophylla Vahl* & marinheiro-do-brejo & zoo & $\mathrm{C}$ & $\mathrm{C}$ & 3753 \\
\hline Trichillia elegans A. Juss. & catiguazinho & zoo & $\mathrm{C}$ & $\mathrm{C}$ & 3754 \\
\hline Trichillia catigua A. Juss. & catiguá & zoo & $\mathrm{C}$ & $\mathrm{C}$ & 3755 \\
\hline Trichillia casaretti C. DC.* & baga-de-morcego & zoo & $\mathrm{C}$ & $\mathrm{C}$ & 3756 \\
\hline Trichillia pallida $\mathrm{Sw}$. & catiguá & zoo & $\mathrm{C}$ & $\mathrm{C}$ & 3758 \\
\hline Monimiaceae & - & - & - & - & - \\
\hline Mollinedia widgrenii A. DC.* & - & zoo & $\mathrm{B}$ & $\mathrm{T}$ & 3758 \\
\hline
\end{tabular}


Tabela 1 (continuação)

\begin{tabular}{|c|c|c|c|c|c|}
\hline Família/ Nome científico & Nome popular & $\mathrm{SD}$ & $\mathrm{TF}$ & GE & No. CBT \\
\hline Mollinedia clavigera Tul.* & pimenteira & zoo & $\mathrm{D}$ & $\mathrm{C}$ & 3759 \\
\hline Moraceae & - & - & - & - & - \\
\hline Ficus guaranitica Chodat. & figueira & zoo & $\mathrm{P}$ & I & 3760 \\
\hline Ficus insipida Willd. & figueira- mata-pau & zoo & $\mathrm{P}$ & I & 3761 \\
\hline Sorocea bonplandii (Baill.) W.C. Burger, Lanj. \& Boer & falsa-espinheira-santa & zoo & $\mathrm{B}$ & $\mathrm{C}$ & 3762 \\
\hline Myrsinaceae & - & - & - & - & - \\
\hline Myrsine loefgrenii (Mez) Otegui* & - & zoo & $\mathrm{D}$ & - & 3763 \\
\hline Myrsine umbellata Mart. & capororocão & zoo & $\mathrm{D}$ & I & 3764 \\
\hline Myrtaceae & - & - & - & - & - \\
\hline Campomanesia xanthocarpa O. Berg & guabiroba & zoo & $\mathrm{B}$ & $\mathrm{T}$ & 3764 \\
\hline Eugenia neoverrucosa (Legr.) Sobral* & - & zoo & $\mathrm{D}$ & $\mathrm{C}$ & 3765 \\
\hline Eugenia ramboi D. Legrand* & - & zoo & $\mathrm{D}$ & $\mathrm{T}$ & 3766 \\
\hline Eugenia uniflora L. & pitanga & zoo & $\mathrm{D}$ & $\mathrm{T}$ & 3768 \\
\hline Myrtaceae sp. $1 *$ & - & zoo & $\mathrm{D}$ & - & 3769 \\
\hline Myrtaceae sp. 2 & jabuticaba & zoo & $\mathrm{D}$ & - & 3770 \\
\hline Nyctaginaceae & - & - & - & - & - \\
\hline Pisonia ambigua Heimerl.* & maria-mole & ane & A & $\mathrm{T}$ & 3771 \\
\hline Bougainvillea spectabilis Willd. & roseira & ane & A & $\mathrm{T}$ & 3772 \\
\hline Phytolaccaceae & - & - & - & - & - \\
\hline Gallesia integrifolia (Spreng.) Harms. & pau-d'alho & ane & $\mathrm{S}$ & $\mathrm{T}$ & 3773 \\
\hline Picramniaceae & - & - & - & - & - \\
\hline Picramnia ramiflora Planch. & camboitá & zoo & $\mathrm{D}$ & $\mathrm{C}$ & 3774 \\
\hline Piperaceae & - & - & - & - & - \\
\hline Piper sp. & - & zoo & $\mathrm{B}$ & $\mathrm{T}$ & 3775 \\
\hline Rhamnaceae & - & - & - & - & - \\
\hline Colubrina glandulosa Perkins & sobrasil & aut & $\mathrm{C}$ & I & 3776 \\
\hline Rosaceae & - & - & - & - & - \\
\hline Prunus myrtifolia (L.) Urb. & pessegueiro-bravo & zoo & $\mathrm{D}$ & I & 3777 \\
\hline Rubiaceae & - & - & - & - & - \\
\hline Alseis floribunda Schott. * & quina-de-são-paulo & ane & $\mathrm{C}$ & I & 3778 \\
\hline Psychotria carthagenensis Jacq.* & café-do-mato & zoo & $\mathrm{D}$ & $\mathrm{T}$ & 3779 \\
\hline Rutaceae & - & - & - & - & - \\
\hline Balfourodendron riedelianum (Engl.) Engl. & pau-marfim & ane & $\mathrm{S}$ & $\mathrm{T}$ & 3780 \\
\hline Esenbeckia grandiflora Mart.* & pau-de-cotia & aut & $\mathrm{C}$ & $\mathrm{T}$ & 3781 \\
\hline Metrodorea nigra A. St.-Hil. & carrapateira & aut & $\mathrm{C}$ & $\mathrm{I}$ & 3782 \\
\hline Pilocarpus pennatifolius Lem. & jaborandi & aut & $\mathrm{C}$ & $\mathrm{C}$ & 3783 \\
\hline Zanthoxylum sp.* & mamica & zoo & $\mathrm{C}$ & $\mathrm{T}$ & 3784 \\
\hline Salicaceae & - & - & - & - & - \\
\hline Casearia gossypiosperma Briq. & pau-de-espeto & ane & $\mathrm{C}$ & $\mathrm{T}$ & 3785 \\
\hline Casearia sylvestris Sw.* & guaçatonga & zoo & $\mathrm{C}$ & I & 3786 \\
\hline Xylosma pseudosalzmanii Sleumer* & espinho-de-judeu & zoo & $\mathrm{B}$ & I & 3787 \\
\hline Sapindaceae & - & - & - & - & - \\
\hline Cupania vernalis Camb. & camboatã & zoo & $\mathrm{C}$ & $\mathrm{T}$ & 3788 \\
\hline Matayba elaeagnoides Radlk.* & camboatã & zoo & $\mathrm{C}$ & $\mathrm{T}$ & 3789 \\
\hline Sapotaceae & - & - & - & - & - \\
\hline Chrysophyllum gonocarpum (Mart. \& Eichler) Engl. & guatambú & zoo & $\mathrm{B}$ & $\mathrm{T}$ & 3790 \\
\hline
\end{tabular}


Tabela 1 (continuação)

\begin{tabular}{|c|c|c|c|c|c|}
\hline Família/ Nome científico & Nome popular & SD & $\mathrm{TF}$ & GE & No. CBT \\
\hline Solanaceae & - & - & - & - & - \\
\hline Cestrum intermedium Sendtn. & coerana & zoo & $\mathrm{B}$ & $\mathrm{I}$ & 3791 \\
\hline Cestrum strigilatum Ruiz \& Pav.* & coerana-de-flor-verde & zoo & $\mathrm{B}$ & $\mathrm{P}$ & 3792 \\
\hline Solanum campaniforme Roem. \& Schult. & - & zoo & $\mathrm{B}$ & $\mathrm{P}$ & 3793 \\
\hline Solanum mauritianum Scop. & fumeiro & zoo & $\mathrm{B}$ & $\mathrm{P}$ & 3794 \\
\hline Urticaceae & - & - & - & - & - \\
\hline Cecropia glaziovi Snethlage* & embaúba-vermelha & zoo & $\mathrm{D}$ & $P$ & 3795 \\
\hline Cecropia pachystacyia Trécul. & embaúba-branca & zoo & $\mathrm{D}$ & $\mathrm{P}$ & 3796 \\
\hline Boehmeria caudata Sw.* & urtiga-mansa & zoo & $\mathrm{D}$ & $\mathrm{P}$ & 3797 \\
\hline Urera baccifera (L.) Gaud. ex Wedd. & urtiga & zoo & $\mathrm{D}$ & $\mathrm{P}$ & 3798 \\
\hline
\end{tabular}

Dentre as espécies arbustivo-arbóreas identificadas, sete espécies de cinco famílias fazem parte da Lista Vermelha de Plantas Ameaçadas de Extinção no Estado do Paraná (Hatschbach \& Ziller 1995): Aspidosperma polyneuron Müll. Arg., Astronium graveolens Jacq., Balfourodendron riedellianum (Engl.) Engl., Casearia gossypiosperma Briq., Centrolobium tomentosum Benth., Lonchocarpus muehlbergianus Hass1. e Myrocarpus frondosus Allemao. Esses resultados expõem a relevância do fragmento para a conservação da biodiversidade, reforçando a importância ecológica da região, apesar do histórico de exploração. Foram encontradas também espécies arbóreas exóticas como limoeiro (Citrus sp.) e santa bárbara (Melia azedarach L.), espécies características de locais perturbados.

Comparando o estudo anterior com o presente levantamento foi observada a mesma sequência de abundâncias das síndromes de dispersão, com maior proporção de espécies zoocóricas no estudo presente (figura 2). Em Tomé et al. (1999) 44\% das espécies encontradas eram zoocóricas (64\% no presente estudo), $27 \%$ anemocóricas, $13 \%$ autocóricas e $16 \%$ não identificadas. Os dados de ambos os estudos estão de acordo com a predição de que a maioria das espécies tropicais apresenta síndrome de dispersão zoocórica (Silva \& Soares-Silva 2000). Esse aumento no número das espécies zoocóricas de acordo com o tempo, embasada pela ideia de que os ambientes de estádios sucessionais intermediários e avançados apresentam comumente maior disponibilidade de frutos zoocóricos (Loiselle \& Blake 1990, Blake \& Loiselle 1991), sugere o avanço sucessional deste fragmento.

As proporções das síndromes de dispersão de sementes encontradas no PEMSF estão de acordo com o encontrado em outro fragmento da região, o Parque Estadual Mata dos Godoy (Silva \& Soares-Silva 2000) que apresenta clima e solo semelhantes, e mesmo tipo vegetacional; bem como ao encontrado na maioria das florestas tropicais e subtropicais, como observados por Howe \& Smalllwood (1982), Spina et al. (2001), Santos \& Kinoshita (2003), Budke et al. (2005), Kinoshita et al. (2006), Giehl et al. (2007) e Yamamoto et al. (2007) (figura 2). Segundo Howe \& Smallwood (1982), de 50\% a 75\% das espécies de árvores desses ecossistemas produzem frutos adaptados à dispersão zoocórica.

Quanto aos tipos de frutos encontrados, o do tipo cápsula foi o mais abundante, sendo característico de uma das famílias que apresentaram maior número de representantes, a família Meliaceae, e também característico da maioria das espécies de Euphorbiaceae (Murillo 2004). O segundo tipo de fruto mais encontrado foi a drupa, que apresenta pericarpo carnoso, característico de síndromes de dispersão zoocórica (Van der Pij1 1982). As espécies pertencentes à família Meliaceae encontradas no estudo, em sua maioria, apresentam fruto do tipo cápsula apresentando também síndrome de dispersão zoocórica, com exceção de Cedrela fissilis Vell. que possui dispersão anemocórica. As espécies Trichilia sp., Guarea macrophylla Vahl e Cabralea canjerana (Vell.) Mart. possuem sementes revestidas de arilo que são expostas após a deiscência do fruto atraindo grande diversidade de aves.

As famílias Lauraceae e Myrtaceae, que apresentam principalmente espécies com frutos do tipo drupa foram encontradas em número de quatro e seis espécies, respectivamente. Segundo Dias et al. (2002) essas famílias aparecem em fragmentos de 


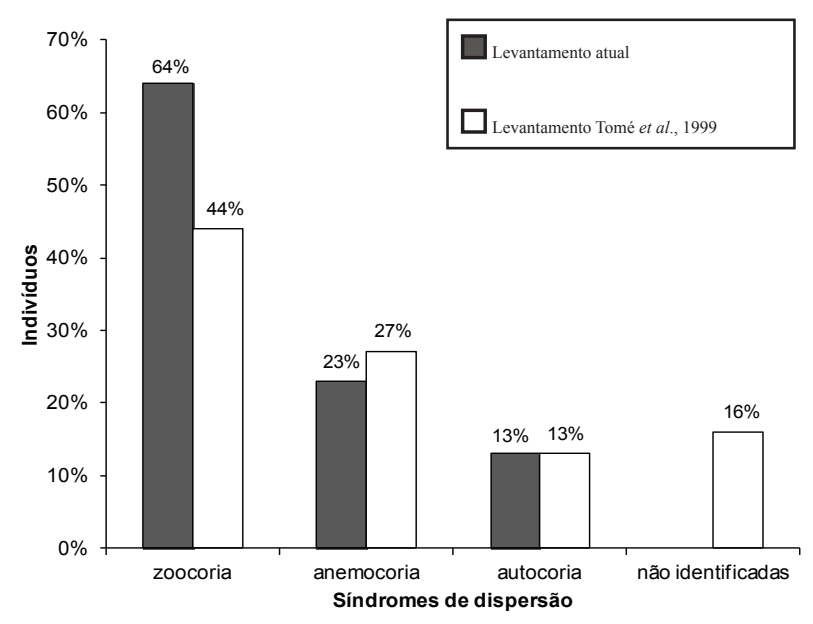

Figura 1: Proporções de espécies zoocóricas, anemocóricas e autocóricas para o total amostrado no Parque Estadual Mata São Francisco, PR, Brasil.

Figure 1: Proportion of zoochoric, anemochoric and autochoric species for all samples in Parque Estadual Mata São Francisco, Paraná State, Brazil.

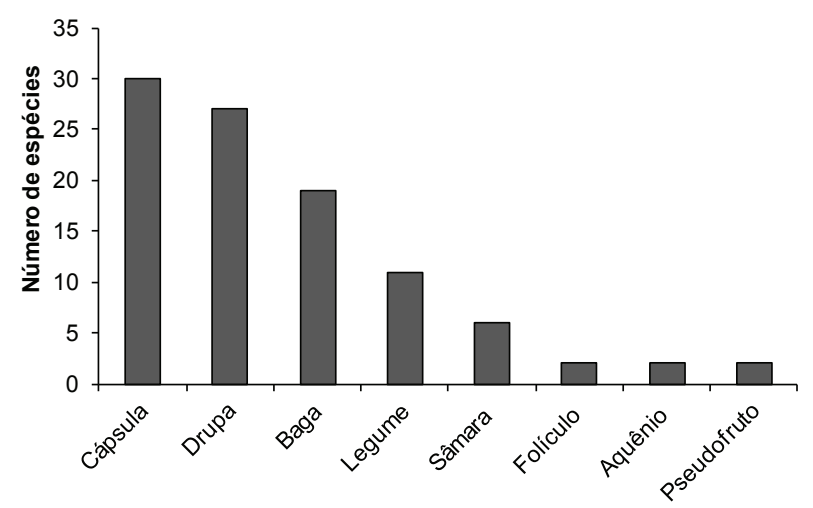

Figura 2: Frequência de frutos em relação à tipologia encontrada no Parque Estadual Mata São Francisco, PR, Brasil.

Figure 2: Frequency of fruits in relation to the type found in São Francisco Forest State Park, PR, Brazil

regiões próximas ao PEMSF, na bacia do rio Tibagi, como duas das famílias com maior riqueza de espécies, sendo importante fonte de alimento para a fauna da região.

A família Myrtaceae tem grande importância para as florestas do sul e sudeste do Brasil, sendo fundamental no processo de recomposição e manutenção da floresta produzindo frutos comestíveis para a avifauna, além de grande produção de pólen que atraem diversos polinizadores (Tomé et al. 1999). Esta é uma das famílias apícolas mais importantes do Brasil (Landrum \& Kawasaki 1997), sendo citada em quase todos os estudos que envolvem a determinação das espécies vegetais de interesse para abelhas. Devido à variada morfologia dos frutos, diferentes grupos de animais funcionam como consumidores e dispersores, sendo que no Brasil aves e primatas são considerados os principais dispersores da família (Gressler et al. 2006).

A família Lauraceae é considerada de grande importância econômica para a Floresta Atlântica em virtude da madeira de boa qualidade, óleos essenciais (Quinet \& Andreata 2002) e por apresentar grande recurso alimentar para diversas espécies de aves que depositam as sementes em diferentes sítios (Krügel et al. 2006). As espécies do gênero Ficus, pertencentes à família Moraceae, também são responsáveis pela alimentação de diversos animais, sendo consideradas espécies-chave, pois produzem sicônios em grande quantidade durante a maior parte do ano, atraindo uma alta diversidade de animais, desde pequenas aves a grandes mamíferos (Reis et al. 1999). Dentre esses animais podem ser citados os quirópteros, predominantemente frugívoros (Fleming 1986), como o Artibeus lituratus (Olfers), espécie encontrada na região (Reis et al. 2002), considerado potencial consumidor e dispersor de frutos de Moraceae (Fleming 1986).

Em levantamento da mastofauna do PEMSF realizado por Rezende (2007) foram encontrados alguns animais, podendo-se citar o bugio - Alouatta clamitans (Cabrera 1940), macaco prego - Cebus nigritus (Goldfuss 1809), lobinho - Cerdocyon thous (Linnaeus 1706), jaguatirica - Leopardus pardalis (Linnaeus 1758), entre outros. Espécies importantes da avifauna também foram observadas por Bornschein \& Reinert (2000), como o fi-fi-verdadeiro - Euphonia chlorotica (Linnaeus 1776) e o tico-tico-do-mato-de-bico-amarelo - Arremon flavirostris (Swainson 1837), espécies que exercem grande função na dispersão de sementes.

Cabe observar que as variadas síndromes de dispersão das espécies arbustivo-arbóreas do presente estudo estão relacionadas com fatores abióticos como clima e solo, e fatores bióticos, ligados à biodiversidade do fragmento. A florística similar observada nos fragmentos próximos denota contínua cobertura vegetal pretérita, o que possibilitava a maior movimentação dos animais e dos propágulos vegetais entre os fragmentos. $\mathrm{O}$ aumento na riqueza, a grande substituição de espécies e a maior proporção de espécies zoocóricas, sugerem o avanço sucessional desse remanescente florestal após o processo de 
exploração. Portanto, a conservação desta área e de áreas similares irá possibilitar a proteção da flora (estritamente ou relacionada com a fauna), e consequentemente, será conservada parte do patrimônio natural brasileiro.

\section{Agradecimentos}

Os autores agradecem ao Instituto Ambiental do Paraná (IAP) por permitir a realização do trabalho na Unidade de Conservação; a todos os funcionários do Parque Estadual Mata São Francisco, em especial a Aparecido Ferreira Dias e Devanil José Bonini. A Edson Mendes Francisco da Universidade Estadual de Londrina (UEL) e a todos do Museu Botânico de Curitiba, entre eles Clarisse Poliquesi, Gerdt Hatschbach, Juarez Cordeiro, Marcos Sobral, Osmar Ribas e outros que contribuíram na identificação de espécies. Ao professor José Antonio Pimenta da UEL, pela disponibilidade e contribuição. Aos assessores anônimos e editores da revista Hoehnea pelas valiosas sugestões. E a todos que participaram da etapa de campo, em especial à Aline Sardinha da Silva, uma das idealizadoras do projeto.

\section{Literatura citada}

APG III. 2009. An update of the Angiosperm Phylogeny Group classification for the orders and families of flowering plants: APG III. Botanical Journal of the Linnean Society 161: 105-121.

Aquino, C. \& Barbosa, L.M. 2009. Classes sucessionais e síndromes de dispersão de espécies arbóreas e arbustivas existentes em vegetação ciliar remanescente (Conchal, $\mathrm{SP}$ ), como subsídio para avaliar o potencial do fragmento como fonte de propágulos para enriquecimento de áreas revegetadas no rio Mogi-Guaçu, SP. Revista Árvore 33: 349-358.

Blake, J.G. \& Loiselle, B.A. 1991. Variation in resource abundance affects capture rates of birds in three lowland habitats in Costa Rica. Auk 108: 114-130.

Bornschein, M.R. \& Reinert, B.L. 2000. Aves de três remanescentes florestais do norte do Estado do Paraná, sul do Brasil, com sugestões para a conservação e manejo. Revista Brasileira de Zoologia 17: 615-636.

Budke, J.C., Athayde, E.A., Giehl, E.L.H., Záchia, R.A. \& Eisinger, S.M. 2005. Composição florística e estratégias de dispersão de espécies lenhosas em uma floresta ribeirinha, arroio Passo das Tropas, Santa Maria, RS, Brasil. Iheringia Série Botânica 60: 17-24.

Budowsky, G. 1965. Distribuition of Tropical American Rain Forest species in the light of sucession process. Turrialba 15: 40-42.
Campassi, F. 2006. Padrões geográficos das síndromes de dispersão e características dos frutos de espécies arbustivo-arbóreas em comunidades vegetais na mata atlântica. Dissertação de Mestrado, Universidade de São Paulo, Piracicaba.

Costa, J.T, Estevan, D.A, Bianchini, E. \& Fonseca, I.C.B. 2011. Composição florística das espécies vasculares e caráter sucessional da flora arbórea de um fragmento de Floresta Estacional Semidecidual no sul do Brasil. Revista Brasileira de Botânica 34: 411-422.

Dias, M.C., Vieira, A.O.S. \& Paiva, M.R.C. 2002. Florística e fitossociologia das espécies arbóreas das florestas da bacia do rio Tibagi. In: M.E. Medri, E.Bianchini, O. Shibata \& J.A. Pimenta (eds.). A Bacia do Rio Tibagi. Ed. UEL, Londrina, pp. 109-124.

Dolibaina, P.C., Silva, S.M. \& Soares-Silva, L.H. 1993. Estudo florístico e fitossociológico do Parque Arthur Thomas, Londrina - PR. In: T.J.A.S. Rêgo (ed.). Anais do $44^{\circ}$ Congresso Nacional de Botânica, São Luís, pp. 306.

Ferri, M.G. 1983. Botânica - Morfologia externa das plantas [organografia]. 15 ed. Nobel, São Paulo.

Fleming, T.H. 1986. Opportunisms versus specialization: evolution of feeding strategies in frugivorous bats. $I n$ : A. Estrada \& T.H. Fleming (eds.). Frugivores and seed dispersal. Dr. W. Junk Publishers, Dordrecht, pp. 105-118.

Fonseca, R.C.B. \& Rodrigues, R.R. 2000. Análise estrutural e aspectos do mosaico sucessional de uma floresta semidecídua em Botucatu, SP. Scientia Forestalis 57: 27-43.

Fundação SOS Mata Atlântica \& INPE. 2008. Atlas dos remanescentes florestais da Mata Attântica período 2000-2005. Fundação SOS Mata Atlântica/Instituto Nacional de Pesquisas Espaciais, São Paulo.

Gandolfi, S. 2000. História natural de uma Floresta Estacional Semidecidual no município de Campinas (São Paulo, Brasil). Tese de Doutorado, Universidade Estadual de Campinas, Campinas.

Giehl, E.L.H., Athayde, E.A., Budke, J.C., Gesing, J.P.A., Einsinger, S.M. \& Canto-Dorow, T.S. 2007. Espectro e distribuição vertical das estratégias de dispersão de diásporos do componente arbóreo em uma floresta estacional no sul do Brasil. Acta Botanica Brasilica 21: 137-145.

Gressler, E., Pizzo, M.A. \& Morellato, L.P.C. 2006. Polinização e dispersão de sementes em Myrtaceae do Brasil. Revista Brasileira de Botânica 29: 509-530.

Hatschbach, G.G. \& Ziller, S.R. 1995. Lista vermelha de plantas ameaçadas de extinção no estado do Paraná. SEMA/GTZ, Curitiba.

Howe, H.F. \& Smallwood, J. 1982. Ecology of seed dispersal. Annual Review of Ecology and Systematics 13: 201-223. 
IAPAR. 2000. Cartas climáticas do Estado do Paraná. http:// www.iapar.br (acesso em 12.08.2008).

Isernhagen, I., Silva, S.M., Rodrigues, W. \& Galvão, F. 2003. A fitossociologia florestal no Paraná: listagem bibliográfica comentada. http://www.ipef.br (acesso em 05.09.2008).

Kinoshita, L.S., Torres, R.B., Martins, E.R.F., Spinelli, T., Ahn, Y.J. \& Constâncio, S.S. 2006. Composição florística e síndromes de polinização e de dispersão da mata do Sítio São Francisco, Campinas, SP, Brasil. Acta Botanica Brasilica 20: 313-327.

Köppen, W. 1948. Climatologia. Fundo de Cultura Econômica, México.

Krügel, M.M., Burger, M.I. \& Alves, M.A. 2006. Frugivoria por aves em Nectandra megapotamica (Lauraceae) em uma área de floresta estacional decidual no Rio Grande do Sul, Brasil. Iheringia Série Zoologia 96: 17-24.

Landrum, L.R. \& Kawasaki, M.L. 1997. The genera of Myrtaceae in Brazil - an illustrated synoptic treatment and identification keys. Brittonia 49: 508-536.

Loiselle, B.A. \& Blake, J.G. 1990. Diets of understory fruit-eating birds in Costa Rica: seasonality and resource abundance. Studies in Avian Biology 13: 91-103.

Mikich, S.B. \& Silva, S.M. 2001. Composição florística e fenologia das espécies zoocóricas no centro-oeste do Paraná. Acta Botanica Brasilica 15: 89-113.

Mueller-Dombois, D. \& Ellenberg, H. 1974. Aims and methods of vegetation ecology. John Wiley, New York.

Murillo, J. 2004. Las Euphorbiaceae de Colombia. Biota Colombia 5: 183-199.

Nathan, R. \& Muller-Landau, H.C. 2000. Spatial patterns of seed dispersal, their determinants and consequences for recruitment. Tree 15: 278-285.

Ouborg, N.J., Piquot, Y. \& Van Groenendael, J.M. 1999. Population genetics, molecular markers and the study of dispersal in plants. Journal of Ecology 87: 551-568.

Quinet, A. \& Andreata, R.H.P. 2002. Lauraceae Jussieu na Reserva Ecológica de Macaé de Cima, município de Nova Friburgo, Rio de Janeiro, Brasil. Rodriguesia 53: 59-121.

Reis, A., Zambonim, R.M. \& Nakazono, E.M. 1999. Reserva da biosfera da Mata Atlântica: Recuperação de áreas florestais degradadas utilizando a sucessão e as interações planta-animal. CETESB - Companhia die Tecnologia Ambiental, São Paulo.

Reis, N.R., Peracchi, A.L. \& Lima, I.P. 2002. Morcegos da bacia do rio Tibagi. In: M.E. Medri, E. Bianchini, O. Shibata \& J.A. Pimenta (eds.). A Bacia do Rio Tibagi. Ed. UEL, Londrina, pp. 251-270.
Rezende, G.Z. 2007. Inventário da mastofauna não voadora de médio e grande porte do Parque Estadual Mata São Francisco. Trabalho de conclusão de curso, Universidade Estadual do Norte do Paraná, Bandeirantes.

Ribeiro, M.C., Metzger, J.P., Martensen, A.C., Ponzoni, F.J. \& Hirota, M.M. 2009. The Brazilian atlantic forest: how much is left, and how is the remaining forest distributed? Implications for conservation. Biological Conservation 142: 1141-1153.

Santos, H.G. 1999. Sistema brasileiro de classificação de solos. Embrapa solos, Rio de Janeiro.

Santos, K. \& Kinoshita, L.S. 2003. Flora arbustivo-arbórea do fragmento de floresta estacional semidecidual do Ribeirão Cachoeira, município de Campinas, SP. Acta Botanica Brasílica 17: 325-341.

Silva, F.C. \& Soares-Silva, L.H. 2000. Arboreal flora of the Godoy Forest State Park, Londrina, PR, Brazil. Edinburgh Journal of Botany 57: 107-120.

Soares-Silva, L.H. \& Barroso, G.M. 1992. Fitossociologia do estrato arbóreo da floresta na porção norte do Parque Estadual Mata dos Godoy, Londrina, PR, Brasil. In: Anais do $8^{\circ}$ Congresso da Sociedade Botânica de São Paulo, Campinas, pp.101-112.

Soares-Silva, L.H., Bianchini, E., Fonseca, E.P., Dias, M.C., Medri, M.E. \& Zangaro Filho, W. 1992. Composição florística e fitossociologia do componente arbóreo das florestas ciliares da bacia do rio Tibagi. 1. Fazenda Doralice, Ibiporã, PR. Revista do Instituto Florestal 4: 199-206.

Spina, A.P., Ferreira, W.M. \& Leitão-Filho, H.F. 2001. Floração, frutificação e síndromes de dispersão de uma comunidade de floresta de brejo na região de Campinas. Acta Botanica Brasilica 15: 349-368.

Tomé, M.V.F. \& Vilhena, A.H. 1996. Composição florística do estrato arbóreo do Parque Estadual Mata São Francisco. In: Anais do $4^{\circ}$ Simpósio Internacional sobre ecossistemas florestais, Belo Horizonte, pp.16-17.

Tomé, M.V.F., Miglioranza, E., Vilhena, A.H. \& Fonseca, E.P. 1999. Composição florística e fitossociológica do Parque Estadual Mata São Francisco. Revista do Instituto Florestal 11: 13-23.

Van der Pijl, L. 1982. Principles of dispersal in higher plants. 3 ed. Springer-Verlag, Berlin.

Veloso, H.P., Rangel-Filho, A.L.R. \& Lima, J.C.A. 1991. Classificação da vegetação brasileira, adaptada a um sistema universal. IBGE, Rio de Janeiro.

Yamamoto, L.F., Kinoshita, L.S. \& Martins, F.R. 2007. Síndromes de polinização e de dispersão em fragmentos da floresta estacional semidecídua montana, SP, Brasil. Acta Botanica Brasilica 21: 553-573. 